\title{
Quorum Quenching Lactonase Strengthens Bacteriophage and Antibiotic Arsenal Against Pseudomonas aeruginosa Clinical Isolates
}

\author{
Sonia Mion ${ }^{1}$, Benjamin Rémy ${ }^{1,2}$, Laure Plener ${ }^{2}$, Fabienne Brégeon ${ }^{1,3}$, Eric Chabrière ${ }^{1 *}$ \\ and David Daudé2* \\ ${ }^{1}$ Aix-Marseille University, IRD, APHM, MEPHI, IHU-Méditerranée Infection, Marseille, France, ${ }^{2}$ Gene\&GreenTK, Marseille, \\ France, ${ }^{3}$ Service des Explorations Fonctionnelles Respiratoires Centre Hospitalo Universitaire Nord, Pôle Cardio-Vasculaire \\ et Thoracique, Assistance Publique des Hôpitaux de Marseille, Marseille, France
}

OPEN ACCESS

Edited by:

Ana Maria Otero,

University of Santiago

de Compostela, Spain

Reviewed by:

Rodolfo García-Contreras, National Autonomous University

of Mexico, Mexico

Jin Zhou,

Graduate School at Shenzhen,

Tsinghua University, China

*Correspondence: Eric Chabrière

eric.chabriere@univ-amu.fr David Daudé

david.daude@gene-greentk.com

Specialty section:

This article was submitted to Antimicrobials, Resistance

and Chemotherapy,

a section of the journal

Frontiers in Microbiology

Received: 23 May 2019

Accepted: 20 August 2019

Published: 03 September 2019

Citation:

Mion S, Rémy B, Plener L, Brégeon F, Chabrière $E$ and Daudé $D$ (2019) Quorum Quenching Lactonase

Strengthens Bacteriophage and Antibiotic Arsenal Against Pseudomonas aeruginosa Clinical Isolates. Front. Microbiol. 10:2049. doi: 10.3389/fmicb.2019.02049
Many bacteria use quorum sensing (QS), a bacterial communication system based on the diffusion and perception of small signaling molecules, to synchronize their behavior in a cell-density dependent manner. QS regulates the expression of many genes associated with virulence factor production and biofilm formation. This latter is known to be involved in antibiotic and phage resistance mechanisms. Therefore, disrupting $\mathrm{QS}$, a strategy known as quorum quenching $(\mathrm{QQ})$, appears to be an interesting way to reduce bacterial virulence and increase antibiotic and phage treatment efficiency. In this study, the ability of the QQ enzyme SsoPox-W263I, a lactonase able to degrade acyl-homoserine lactones, was investigated for quenching both virulence and biofilm formation in clinical isolates of Pseudomonas aeruginosa from diabetic foot ulcers, as well as in the PA14 model strain. These strains were further evolved to resist to bacteriophage cocktails. Overall, 10 antibiotics or bacteriophage resistant strains were evaluated and SsoPox-W263I was shown to decrease pyocyanin, protease and elastase production in all strains. Furthermore, a reduction of more than $70 \%$ of biofilm formation was achieved in six out of ten strains. This anti-virulence potential was confirmed in vivo using an amoeba infection model, showing enhanced susceptibility toward amoeba of nine out of ten $P$. aeruginosa isolates upon $Q Q$. This amoeba model was further used to demonstrate the ability of SsoPox-W263I to enhance the susceptibility of sensitive and phage resistant bacteria to bacteriophage and antibiotic.

Keywords: quorum quenching, quorum sensing, lactonase, AHL, Pseudomonas aeruginosa, multidrug resistant bacteria, antibiotics, bacteriophages

\section{INTRODUCTION}

Pseudomonas aeruginosa is an opportunistic human pathogen involved in numerous diseases from otitis to keratitis, wound and burn infections, pneumonia and urinary tract infections (Driscoll et al., 2007). P. aeruginosa isolates are among the most frequently found antibiotic-resistant pathogens involved in diabetic foot infections, their presence is usually associated with morbidity 
(Ertugrul et al., 2012). Surveillance of $P$. aeruginosa infections has revealed trends of increasing resistance to antibiotic treatments (Breidenstein et al., 2011) and the priority to support research and development of effective drugs against antibiotic-resistant $P$. aeruginosa was recently defined as critical by the World Health Organization (WHO) (Tacconelli et al., 2018).

This last decade, bacteriophage (phage) therapy has regained interest as a new weapon to treat antibiotic-resistant infections (Rolain et al., 2015; Domingo-Calap et al., 2016) and is under consideration to treat $P$. aeruginosa infections. Phages are the most abundant predator of bacteria in nature (Suttle, 2005) and have been domesticated, especially in Eastern Europe, to treat enteric infections, such as cystic fibrosis (Rolain et al., 2015), dysentery (Salmond and Fineran, 2015), diabetic foot infection, chronic osteomyelitis and other surgical and wound infections (Kutter et al., 2010). Phages offer the advantage of specifically targeting their host bacteria by being harmless to the commensal flora (Loc-Carrillo and Abedon, 2011) and human cells (Domingo-Calap et al., 2016). In western countries, clinical trials have been conducted to assay the therapeutic potential of phages (Rhoads et al., 2009; Wright et al., 2009; Kakasis and Panitsa, 2018), for example to treat $P$. aeruginosa infected burns (Jault et al., 2018). However, as for antibiotics, phages suffer resistance phenomena that may hinder the development of bacteriophage-based therapy (Jault et al., 2018). Finding new therapeutic strategies to limit bacterial resistance is thus of great interest.

In this way, another alternative to treat $P$. aeruginosa infections aims to highjack its communication system referred to as quorum sensing (QS). In P. aeruginosa, QS mainly relies on the secretion and perception of $N$-acyl homoserine lactones (AHL) to orchestrate its behavior, including virulence and biofilm formation as well as the CRISPR-Cas defense system, in a celldensity dependent manner (Bassler and Losick, 2006; HøylandKroghsbo et al., 2017). Interconnections between bacterial QS and susceptibility to bacteriophage infections were also identified (Qin et al., 2017; Saucedo-Mora et al., 2017). Disrupting QS, a strategy referred to as quorum quenching (QQ), is highly attractive to counteract bacterial virulence by using QS inhibitors (QSI) or QQ enzymes (QQE). Among QQE, special attention has been paid to the robust lactonase SsoPox-W263I (Hiblot et al., 2013) that was proved to efficiently inhibit virulence in vitro in model and clinical strains of $P$. aeruginosa (Guendouze et al., 2017) and to drastically decrease mortality in a rat pulmonary infection model (Hraiech et al., 2014). Furthermore, the impact of SsoPox-W263I on CRISPR-Cas gene expression of $P$. aeruginosa was recently demonstrated in both model and clinical strains suggesting that enzymatic QQ may modulate bacterial susceptibility to bacteriophages (Mion et al., 2019).

Here, we consider the use of SsoPox-W263I in clinical isolates of $P$. aeruginosa from diabetic foot ulcers together with the model strain PA14 and further evolved these strains to resist bacteriophages. Knowing that the evolutionary selection of phage resistance in bacteria can induce phenotypic shifts (Labrie et al., 2010), we focused our interest on determining the potential of QQ to control antibiotic and phage-resistant bacteria. Our results show that SsoPox-W263I is efficient to decrease virulence or biofilm in these multi-resistant strains, both in vitro and in vivo using an amoeba model. In the last part, we investigate the QQE treatment to enhance the therapeutic effect of antibiotic and phage when used as a co-treatment in the amoeba infection model. This study shows that QQ is an interesting strategy to treat bacterial infections that can be used to strengthen the effect of antimicrobial treatments.

\section{MATERIALS AND METHODS}

\section{Bacterial Strains and Growth Conditions}

Experiments were conducted using model strain PA14 and three clinical isolates of $P$. aeruginosa, isolated from diabetic patients of the Nimes University Hospital presenting diabetic foot infections. All the patients received an oral information, were anonymized and gave a non-opposition statement to bacterial storage. This study was approved by the local ethics committee (South Mediterranean III) and was carried out in accordance with the Declaration of Helsinki as revised in 2008. Clinical isolates of $P$. aeruginosa and model strain PA14 (UCBPP-PA14) were inoculated from a single colony and pre-cultivated during $6 \mathrm{~h}$ at $37^{\circ} \mathrm{C}$ in Luria Bertani (LB) medium $\left(10 \mathrm{~g} \mathrm{l}^{-1} \mathrm{NaCl}, 10 \mathrm{~g} \mathrm{l}^{-1}\right.$ Tryptone, $5 \mathrm{~g} \mathrm{l}^{-1}$ yeast extract) with agitation at $650 \mathrm{rpm}$. Then, precultures were diluted by a 1,000 factor in MOPS minimal medium complemented with nitrogen $\left(15 \mathrm{mM} \mathrm{NH}_{4} \mathrm{Cl}\right)$, iron $(5 \mu \mathrm{M}$ $\left.\mathrm{Fe}_{2} \mathrm{SO}_{4}\right)$, phosphate $\left(4 \mathrm{mM} \mathrm{K} \mathrm{HPO}_{4}\right)$ and glutamate $(25 \mathrm{mM})$ as carbon source (MOPS glutamate) (Welsh and Blackwell, 2016) and cultures were incubated at $37^{\circ} \mathrm{C}$ under agitation at $650 \mathrm{rpm}$. Enzymes were added, when indicated, at $0.5 \mathrm{mg} \mathrm{ml}^{-1}$. For virulence factor and biofilm formation analysis cultures were incubated during $20 \mathrm{~h}$.

\section{Phage Production and Isolation}

A bacteriophage cocktail (Intesti-bacteriophage, Microgen, Russia) was used for this study. Isolated phage $\Phi$ Intesti-PA14 corresponds to the isolation and concentration of plaque-forming unit (PFU) formed by the phage cocktail on P. aeruginosa PA14 according to the following protocol.

The double agar overlay plaque assay was used to determine the phage titer and isolate phages from phage cocktail (Kropinski et al., 2009). $500 \mu \mathrm{l}$ of an overnight culture of bacteria was added to $4.5 \mathrm{ml}$ of molten soft LB-agar $(0.75 \%)$ and overlaid onto a hard LB-agar plate. Once dry, $10 \mu \mathrm{l}$ drops of phage cocktail were spotted on the soft LB-agar layer. Plates were incubated overnight at $37^{\circ} \mathrm{C}$. Lytic plaques were collected and suspended in $500 \mu \mathrm{l}$ of $\mathrm{MgSO}_{4}(10 \mathrm{mM})$. After chloroform treatment and $10 \mathrm{~min}$ of centrifugation at $4,500 \mathrm{~g}$, the supernatant was filtered at $0.22 \mu \mathrm{m}$. The resulting phage suspension was again spotted on a double-layer plate, and the experiment was repeated until a $10^{8} \mathrm{PFU} \mathrm{m} \mathrm{m}^{-1}$ suspension was obtained. To estimate the phage titer, serial dilutions (from $10^{0}$ to $10^{-10}$ ) of phage suspension were performed in $\mathrm{MgSO}_{4}(10 \mathrm{mM})$, then $10 \mu \mathrm{l}$ of each dilution was spotted on the double-layer plate. The plate was incubated overnight at $37^{\circ} \mathrm{C}$. The titer (PFU ml ${ }^{-1}$ ) was determined by the calculation of lytic PFU for $1 \mathrm{ml}$ of phage suspension. 


\section{Isolation of Phage Resistant $P$. aeruginosa Bacteria}

Precultures were performed by inoculating one colony of the initial strains in LB medium during 6-8 $\mathrm{h}$, then the precultures were diluted by a 10,000 factor in MOPS glutamate, $3 \mathrm{ml}$ were transferred in each well of a 12-well plate. 10, 50 , or $100 \mu \mathrm{l}$ of bacteriophage containing cocktail (Intestibacteriophage, İicrogen, Russia) were added to each well. The plate was incubated at $37^{\circ} \mathrm{C}$ overnight under agitation at $650 \mathrm{rpm}$. The OD $600 \mathrm{~nm}$ was then measured using a plate reader (Synergy HT, BioTek). Cultures showing complete lysis were diluted in a solution of phosphate saline buffer (PBS) and 20\% glycerol. The dilutions were then plated on LB agar. Plates were incubated at $37^{\circ} \mathrm{C}$ overnight.

After $16 \mathrm{~h}$ of incubation, the isolated colonies were picked, cultivated in LB medium and stocked in $20 \%$ glycerol at $-150^{\circ} \mathrm{C}$. The tolerance of the isolated bacteria to the phage cocktail was verified by exposing the strains to the same conditions as those used for their isolation and by verifying that the final OD $600 \mathrm{~nm}$ was indeed higher for the resistant strain than for the parental strain.

\section{Protein Production and Purification}

Two SsoPox variants namely W263I and 5A8 were used as QQ and inactive enzymes, respectively. Productions were realized as previously described (Hiblot et al., 2012, 2013; Hraiech et al., 2014). Briefly, Escherichia coli BL21 (DE3)-pGro7/GroEL cells (TaKaRa), carrying plasmid pET22b-SsoPox-W263I or pET22b-SsoPox-5A8, were cultivated in ZYP-5052 medium complemented with $100 \mu \mathrm{g} \mathrm{ml}^{-1}$ ampicillin and $34 \mu \mathrm{g} \mathrm{ml}^{-1}$ chloramphenicol at $37^{\circ} \mathrm{C}$ until $\mathrm{OD} 600 \mathrm{~nm}$ reached $0.8-1$. The expression of chaperone proteins was induced by adding Larabinose at a final concentration of $0.2 \%(\mathrm{w} / \mathrm{v})$. At the same time, the temperature was reduced to $23^{\circ} \mathrm{C}$ and $0.2 \mathrm{mM}$ of $\mathrm{CoCl}_{2}$ was added. After $20 \mathrm{~h}$ of incubation, the cells were harvested by centrifugation $\left(4,400 \mathrm{~g}, 4^{\circ} \mathrm{C}, 20 \mathrm{~min}\right)$, the pellet was resuspended in lysis buffer [50 mM HEPES pH 8, $150 \mathrm{mM}$ $\mathrm{NaCl}, 0.25 \mathrm{mg} \mathrm{ml}^{-1}$ lysozyme, $0.1 \mathrm{mM}$ Phenylmethylsufonyl fluoride (PMSF) and $10 \mathrm{mg} \mathrm{ml}^{-1}$ DNaseI] and stored at $-80^{\circ} \mathrm{C}$ during $16 \mathrm{~h}$. Frozen cells were thawed at $37^{\circ} \mathrm{C}$ during $15 \mathrm{~min}$ and lysed by three steps of $30 \mathrm{~s}$ sonication (QSonica sonicator Q700, amplitude at 45). Cell debris were removed by centrifugation $\left(21,000 \mathrm{~g}, 4^{\circ} \mathrm{C}, 15 \mathrm{~min}\right)$. Crude extract was incubated during $30 \mathrm{~min}$ at $80^{\circ} \mathrm{C}$ and then centrifuged to precipitate $E$. coli proteins $\left(21,000 \mathrm{~g}, 4^{\circ} \mathrm{C}, 30 \mathrm{~min}\right)$. The enzyme was then concentrated by overnight incubation at $4^{\circ} \mathrm{C}$ in $75 \%$ ammonium sulfate. After resuspension in activity buffer $(50 \mathrm{mM}$ HEPES pH 8, $150 \mathrm{mM} \mathrm{NaCl}, 20.2 \mathrm{mM} \mathrm{CoCl} 2$ ) ammonium sulfate was eliminated by desalting (HiPrep 26/10 desalting, GE Healthcare, ÄKTA Avant). The protein sample obtained was concentrated to $2 \mathrm{ml}$ and then loaded on a size-exclusion chromatography column and purified to homogeneity (HiLoad 16/600 Superdex ${ }^{\mathrm{TM}} 75 \mathrm{pg}$, GE Healthcare, ÄKTA Avant). Protein purity was checked by migration on 10\% SDS-PAGE and protein concentration was measured using a spectrophotometer NanoDrop 2000 (Thermo Scientific).

\section{Antibiograms}

Antibiotic sensitivity of the strains was determined on a Mueller Hinton agar (BioMerieux). The disk diffusion method was realized using the following antibiotics: amikacin $(30 \mu \mathrm{g})$, cefepime $(30 \mu \mathrm{g})$, ceftazidime $(30 \mu \mathrm{g})$, ciprofloxacin $(5 \mu \mathrm{g})$, doxycycline $(30 \mu \mathrm{g})$, fosfomycin $(50 \mu \mathrm{g})$, imipenem $(10 \mu \mathrm{g})$, nitrofurantoin $(300 \mu \mathrm{g})$, piperacillin/tazobactam $(85 \mu \mathrm{g})$, ticarcillin $(75 \mu \mathrm{g})$, ticarcillin/clavulanate $(85 \mu \mathrm{g})$, trimethoprim/sulfamethoxazole $(25 \mu \mathrm{g})$, tobramycin $(10 \mu \mathrm{g})$, rifampicin $(30 \mu \mathrm{g})$. The results were interpreted according to the EUCAST guidelines (European Society of Clinical Microbiology and Infectious Diseases, 2018 $)$ using the Scan ${ }^{\circledR}$ 1200 (Interscience) (Diop et al., 2016).

\section{Analysis of Virulence Factor Production}

Virulence factor productions for the different strains were determined in vitro after a 20 -h culture in presence of $0.5 \mathrm{mg} \mathrm{ml}^{-1}$ SsoPox-W263I or inactive mutant SsoPox$5 \mathrm{~A} 8$ as control.

\section{Pyocyanin Production}

Cell-free culture supernatants were prepared by centrifugation for $5 \mathrm{~min}$ at 12,000 $\mathrm{g}$. Pyocyanin was extracted by mixing $500 \mu \mathrm{l}$ of cell-free supernatant with $250 \mu \mathrm{l}$ of chloroform (Price-Whelan et al., 2007). After vortexing and $5 \mathrm{~min}$ of centrifugation at $12,000 \mathrm{~g}, 200 \mu \mathrm{l}$ of the bottom chloroform phase were transferred into a quartz 96-well plate. The absorbance was measured at $690 \mathrm{~nm}$ using a plate reader (Synergy HT, BioTek).

\section{Proteolytic Activity}

The protease activity was measured using azocasein (Sigma) (Chessa et al., 2000). $675 \mu \mathrm{l}$ of PBS solution pH 7 were mixed with $50 \mu \mathrm{l}$ of azocasein $\left(30 \mathrm{mg} \mathrm{ml}^{-1}\right.$ in water) and $25 \mu \mathrm{l}$ of cellfree supernatant. After $2 \mathrm{~h}$ of incubation at $37^{\circ} \mathrm{C}, 125 \mu \mathrm{l}$ of $20 \%$ $(\mathrm{w} / \mathrm{v})$ trichloroacetic acid were added to stop the reaction. The solution was then centrifugated for $10 \mathrm{~min}$ at $10,000 \mathrm{~g}$ and the absorbance of $200 \mu \mathrm{l}$ of the supernatant was measured at $366 \mathrm{~nm}$ using a plate reader (Synergy HT, BioTek).

\section{Elastolytic Activity}

Elastase B activity was measured using elastin-Congo red conjugate (Sigma) degradation assay (Smith et al., 2003). $50 \mu$ l of cell-free supernatant were added to $150 \mu \mathrm{l}$ of elastin-Congo red solution $\left(5 \mathrm{mg} \mathrm{ml}^{-1}\right.$ in $10 \mathrm{mM}$ Tris- $\mathrm{HCl}$ and $1 \mathrm{mM} \mathrm{CaCl}_{2}$ buffer at $\mathrm{pH}$ 7.2) into a 96-well plate. The plate was then sealed with an aluminum membrane and incubated during $24 \mathrm{~h}$ at $37^{\circ} \mathrm{C}$ under agitation $(300 \mathrm{rpm})$. After sedimentation of undigested elastinCongo red conjugate $100 \mu \mathrm{l}$ of the upper phase was transferred to an empty well and the absorbance was measured at $490 \mathrm{~nm}$ using a plate reader (Synergy HT, BioTek).

\section{Biofilm Formation}

The biofilm formed in each well was quantified using crystal violet (Sigma) staining as previously described (Stepanović et al., 2000). Briefly, planktonic cells (non-attached) were first removed

\footnotetext{
${ }^{1}$ http://www.eucast.org/clinical_breakpoints/
} 
by washing the wells with $4 \mathrm{ml}$ of PBS. The plates were then dried at $37^{\circ} \mathrm{C}$ and the biofilm was stained by adding $4 \mathrm{ml}$ of crystal violet $0.05 \%$, incubated for $3 \mathrm{~min}$ under agitation at $150 \mathrm{rpm}$. Then, the crystal violet was removed and each well was rinsed with $4 \mathrm{ml}$ of PBS. Crystal violet was then resolubilized by adding $3 \mathrm{ml}$ of ethanol 96\%. $200 \mu \mathrm{l}$ of the solution was transferred to a 96-well plate and the final concentration of crystal violet was measured at OD $595 \mathrm{~nm}$ using a plate reader (Synergy HT, BioTek).

\section{Virulence Assay Toward Amoeba}

In vivo virulence assay was adapted from a previously described procedure using $P$. aeruginosa and Acanthamoeba polyphaga Linc AP1 (Fenner et al., 2006). Briefly, $3 \mathrm{ml}$ of bacterial culture were pelleted down and resuspended in Page's amoeba saline (PAS) buffer $\left(2 \mathrm{mM} \mathrm{NaCl}, 16 \mu \mathrm{M} \mathrm{MgSO}_{4}, 27 \mu \mathrm{M} \mathrm{CaCl}_{2}, 0.53 \mathrm{mM}\right.$ $\mathrm{Na}_{2} \mathrm{HPO}_{4}, 1 \mathrm{mM} \mathrm{KH} \mathrm{PO}_{4}, \mathrm{pH}$ 6.9) after a culture in 6-well plates (Nunc ${ }^{\mathrm{TM}}$, Thermo Scientific). A. polyphaga Linc AP1 was cultivated during 2-3 days into peptone yeast extract glucose (20 $\mathrm{g} \mathrm{l}^{-1}$ proteose peptone, $2 \mathrm{~g} \mathrm{l}^{-1}$ yeast extract, $0.1 \mathrm{M}$ glucose, $4 \mathrm{mM} \mathrm{MgSO}_{4}, 0.53 \mathrm{mM} \mathrm{CaCl}_{2}, 3.4 \mathrm{mM}$ sodium citrate, $50 \mu \mathrm{M}$ $\left(\mathrm{NH}_{4}\right)_{2} \mathrm{Fe}\left(\mathrm{SO}_{4}\right)_{2}, 2.5 \mathrm{mM} \mathrm{KH}{ }_{2} \mathrm{PO}_{4}, 1.3 \mathrm{mM} \mathrm{Na} \mathrm{HPO}_{4}, \mathrm{pH}$ 6.8) medium at $28^{\circ} \mathrm{C}$ (Fenner et al., 2006). Amoeba cells were recovered after centrifugation at $750 \mathrm{~g}$ and resuspended into PAS buffer to $10^{5}$ cells $\mu \mathrm{l}^{-1}$. Then, $1 \mathrm{ml}$ of bacterial suspension was spread on a PAS agar plate and was left to dry at room temperature. At the center of each plate, $5 \mu \mathrm{l}$ of $A$. polyphaga were spotted and dried at room temperature. Then, plates were incubated at $30^{\circ} \mathrm{C}$ over 7 days and amoeba propagation was followed by directly measuring the central spot with a ruler.

To test the combinatory effect of enzymatic and antibiotic treatments, the MOPS bacterial culture was treated with $10 \mu \mathrm{g} \mathrm{ml}^{-1}$ of enzyme (either SsoPox-W263I or inactive variant SsoPox-5A8 as control) and $25 \mu \mathrm{g} \mathrm{ml}^{-1}$ ciprofloxacin was added to PAS buffer during the resuspension step.

To test the combinatory effect of enzymatic and phage treatments, the MOPS bacterial culture was treated with $10 \mu \mathrm{g} \mathrm{ml}^{-1}$ of enzyme (either SsoPox-W263I or inactive variant SsoPox-5A 8 as control) and $10^{7} \mathrm{PFU} \mathrm{ml^{-1 }}$ of Intesti-PA14 phage was added to PAS buffer during the resuspension step.

\section{RESULTS}

\section{Evaluating Sensitivity of Clinical Isolates to Antibiotics and Bacteriophage Cocktail}

PA14 is a model strain originally isolated from a burn wound (Soyza et al., 2013) and B10, C5, and C11 were isolated from diabetic foot ulcerations (Guendouze et al., 2017). Antibiotic susceptibility of the strains was evaluated using the disk diffusion method with 14 antibiotics and analyzed according the EUCAST recommendations (Figure 1A). The strains were non-susceptible (resistant or intermediate) to at least two different antibiotics tested belonging to rifamycin, sulfonamide or nitrofuran classes. All strains were found sensitive to the tested agents in $\beta$-lactam, aminoglycosides and fosfomycin antimicrobial classes which are commonly used to fight pseudomonal infections. In addition to antibiotic sensitivity, the impact of a commercial bacteriophage cocktail on the strains was evaluated. Interestingly, all the strains were sensitive to the cocktail resulting in drastic decreases in cell density (Supplementary Figure 1). These results confirm that bacteriophage-based therapy may constitute an alternative to antibiotherapies in case of resistant infections.

\section{Isolation and Characterization of Phage-Resistant Variants}

Although bacteriophages were virulent to all four strains, resistance phenomena were rapidly observed after exposure of PA14, B10, C5, and C11 to three different concentrations of phage cocktail for $16 \mathrm{~h}$. For PA14, B10, and C11 one mutant was isolated from each strain: PA14R1, B10R1, and C11R1, respectively. Three different mutants, presenting different phenotypes, were isolated from C5: C5R1, C5R2, and C5R3. The newly isolated mutants were cultured in the presence of the same amount of phage cocktail as that used for their isolation to confirm their resistance (i.e., $100 \mu \mathrm{l}$ for PA14 and PA14R1, $50 \mu \mathrm{l}$ for $\mathrm{B} 10$ and B10R1 and $10 \mu \mathrm{l}$ for C5, C5R1, C5R2, C5R3, C11, and C11R1). Cell density was compared to the initial strains in the presence of phages after a 16-h culture by measuring the OD $600 \mathrm{~nm}$ (Figure 2). In the presence of phages, growth was 4 to 7 times higher for all mutants than for parental strains (Figure 2). The resistance of the isolated mutants against the phage cocktail was thereby clearly highlighted (Figure 2). Antibiotic sensitivity patterns of the phage-resistant strains were further evaluated (Figure 1B). As for parental isolates, phage resistant strains were non-susceptible (resistant or intermediate) to at least two different antibiotics tested belonging to rifamycin, sulfonamide or nitrofuran classes. Phage-resistant strains were found to be sensitive to the tested agents in $\beta$-lactam, aminoglycosides, cephalosporins, carbapenems and fosfomycin antimicrobial classes. As noticed for B10, B10R1 showed intermediate resistance to rifampicin while all other strains were resistant to this antibiotic. Interestingly, the acquisition of bacteriophage resistance was detrimental to antibiotic resistance in the resistant clones isolated from C5. C5R1, C5R2, and C5R3 lost their resistance against doxycycline and ciprofloxacin, C5R1 being also sensitive to nitrofurantoin conversely to C5, C5R2, or C5R3. Similarly, C11R1 exhibited a lower tolerance to trimethoprim/sulfamethoxazole and doxycycline than C11.

\section{Quenching Virulence Factors of Antibiotic or Phage Resistant Clones in vitro}

The QQ effect of SsoPox-W263I on P. aeruginosa isolates PA14, $\mathrm{C} 5, \mathrm{C} 11, \mathrm{~B} 10$ and their bacteriophage resistant counterparts was investigated by measuring the production of three typical virulence factors in vitro: pyocyanin, protease, elastase as well as biofilm production. Under the tested conditions, the addition of SsoPox-W263I significantly reduced the three virulence factors for all strains compared to controls (Figures 3A-C). All tested strains produced detectable levels of pyocyanin and elastolytic 


\begin{tabular}{lcccc} 
A & \multicolumn{4}{c}{ Clinical isolates } \\
\hline Antibiotics & PA14 & B10 & C5 & C11 \\
\hline TIC $(75 \mu \mathrm{g})$ & 25.8 & 24.2 & 21.5 & 19.6 \\
TCC $(85 \mu \mathrm{g})$ & 24.4 & 23.6 & 19.4 & 24 \\
TZP $(85 \mu \mathrm{g})$ & 29.5 & 28.6 & 25.6 & 26 \\
CAZ $(30 \mu \mathrm{g})$ & 28.6 & 26.9 & 25.5 & 24.6 \\
FEP $(30 \mu \mathrm{g})$ & 30.4 & 28.4 & 26.6 & 25.6 \\
IPM $(10 \mu \mathrm{g})$ & 32.7 & 26.1 & 31.7 & 26.5 \\
RA $(30 \mu \mathrm{g})$ & 0 & 16.8 & 11.4 & 9.9 \\
FF $(50 \mu \mathrm{g})$ & 25.3 & 19 & 21.1 & 18.6 \\
SXT $(25 \mu \mathrm{g})$ & 12 & 10 & 13.5 & 0 \\
F $(300 \mu \mathrm{gg})$ & 0 & 11.2 & 7.6 & 0 \\
TM $(10 \mu \mathrm{g})$ & 26.1 & 21.7 & 23.4 & 20.3 \\
AN $(30 \mu \mathrm{g})$ & 28.7 & 21.8 & 23.7 & 20.3 \\
DO $(30 \mu \mathrm{g})$ & 19.4 & 14.1 & 10.3 & 11.4 \\
CIP $(5 \mu \mathrm{g})$ & 36.8 & 33.2 & 19.1 & 33.2 \\
\hline
\end{tabular}

\begin{tabular}{lcccccc} 
B & \multicolumn{7}{c}{ Phage resistant isolates } \\
\hline Antibiotics & PA14R1 & B10R1 & C5R1 & C5R2 & C5R3 & C11R1 \\
\hline TIC $(75 \mu \mathrm{g})$ & 25.2 & 24.2 & 23.2 & 24.1 & 25.6 & 24.5 \\
TCC $(85 \mu \mathrm{g})$ & 22.8 & 23.9 & 25 & 25.3 & 26.2 & 25.3 \\
TZP $(85 \mu \mathrm{g})$ & 31.1 & 26.5 & 31.6 & 30.8 & 34.4 & 29.2 \\
CAZ $(30 \mu \mathrm{g})$ & 31.4 & 25.8 & 31.1 & 30.4 & 30.3 & 29.9 \\
FEP $(30 \mu \mathrm{g})$ & 32.2 & 27 & 33.8 & 32.6 & 32.2 & 30.6 \\
IPM $(10 \mu \mathrm{g})$ & 33.1 & 25.7 & 28.1 & 32.9 & 33.9 & 32.2 \\
RA $(30 \mu \mathrm{g})$ & 8.9 & 12.6 & 9.7 & 9.9 & 12.3 & 10 \\
FF $(50 \mu \mathrm{g})$ & 25.1 & 18 & 24.7 & 24.2 & 25.5 & 26.5 \\
SXT $(25 \mu \mathrm{g})$ & 10.2 & 12.7 & 10.5 & 7.6 & 11.8 & 12.3 \\
F $(300 \mu \mathrm{g})$ & 9 & 11.2 & 16.9 & 9.5 & 9.8 & 7.2 \\
TM $(10 \mu \mathrm{g})$ & 27.2 & 21.3 & 26.1 & 26.2 & 26 & 25.4 \\
AN $(30 \mu \mathrm{g})$ & 29.7 & 20.6 & 30 & 29.7 & 30 & 29.2 \\
DO $(30 \mu \mathrm{g})$ & 19.6 & 11.9 & 19.8 & 20.3 & 19.2 & 17.3 \\
CIP $(5 \mu \mathrm{g})$ & 43.2 & 33.6 & 35.2 & 36.3 & 36.4 & 36.1 \\
\hline
\end{tabular}

Susceptibility expressed as: Resistant, red; Intermediate, orange; Sensitive, green. TIC: Ticarcillin, TCC:

Ticarcillin/Clavulanate, TZP: Piperacillin/Tazobactam, CAZ: Ceftazidime, FEP: Cefepime, IPM: Imipenem, RA: Rifampicin, FF: Fosfomycin, SXT: Trimethoprim/Sulfamethoxazole, F: Nitrofurantoin, TM: Tobramycin, AN: Amikacin, DO: Doxycycline, CIP: Ciprofloxacin

FIGURE 1 | Interpretative zone diameters ( $\mathrm{mm}$ ) of 14 antibiotics used on clinical isolates of Pseudomonas aeruginosa (A) and their associated phage-resistant mutants (B). Susceptibility is expressed as Resistant (red), Intermediate (orange), Sensitive (green).

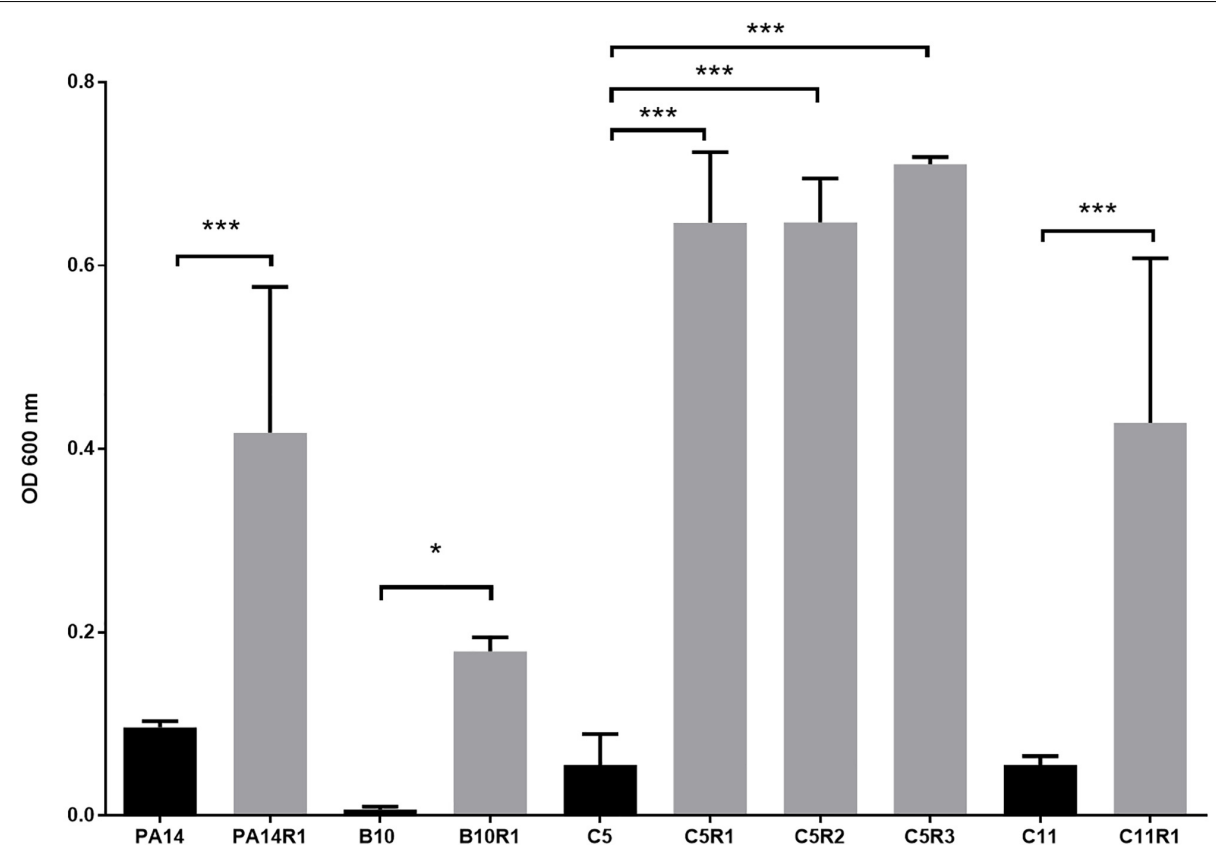

FIGURE 2 | Growth of phage resistant (gray) and the associated parental strains (black) in presence of phage cocktail. For each strain, bars represent the mean density $(\mathrm{OD} 600 \mathrm{~nm})$ after $16 \mathrm{~h}$ of incubation in MOPS glutamate with phage cocktail. Error bars represent the standard deviations of three replicated experiments. ${ }^{*} p$-values $<0.05 ;{ }^{* * *} p$-values $<0.001$ according to Student's $t$-test or ANOVA analysis.

activity. Pyocyanin levels were reduced by more than $75 \%$ in all strains upon enzymatic treatment, while elastase levels were reduced by at least $60 \%$ in nine out of ten strains with the addition of SsoPox-W263I (C5 elastase level being reduced by only $25 \%$ ). Proteolytic activity was detectable for all tested strains but one: the parental isolate $\mathrm{C} 5$ which did not produce sufficient levels of proteases with or without SsoPox-W263I treatment to be detected. Conversely, the three phage-resistant derivatives obtained from C5 had each detectable protease activity in the absence of enzyme and this activity was completely extinguished by the addition of SsoPox-W263I (Figure 3A). Biofilm production was significantly reduced by more than $70 \%$ in six strains when treated with SsoPox-W263I (Figure 3D). Interestingly, the production of these four factors differed 


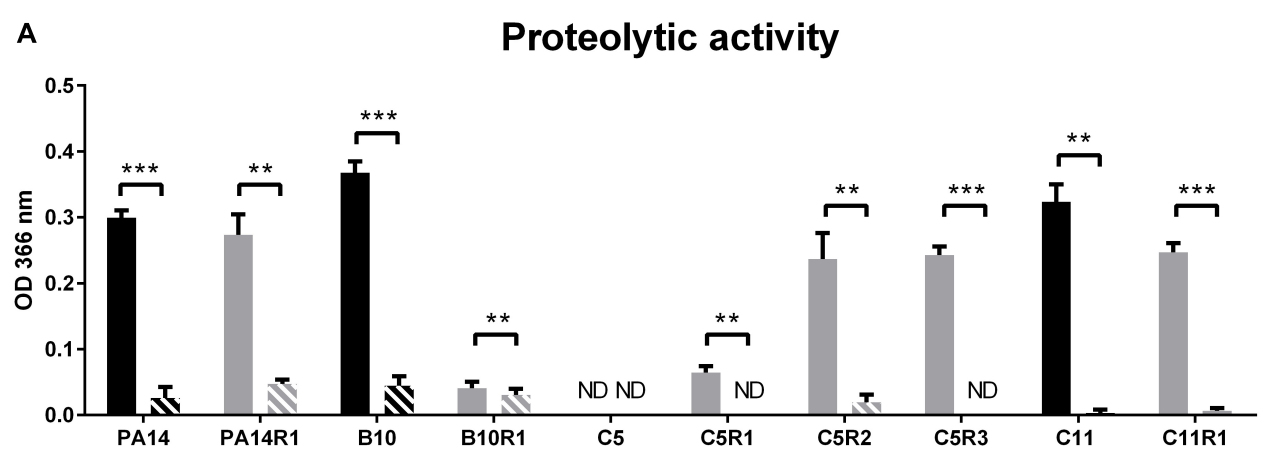

B

Elastolytic activity

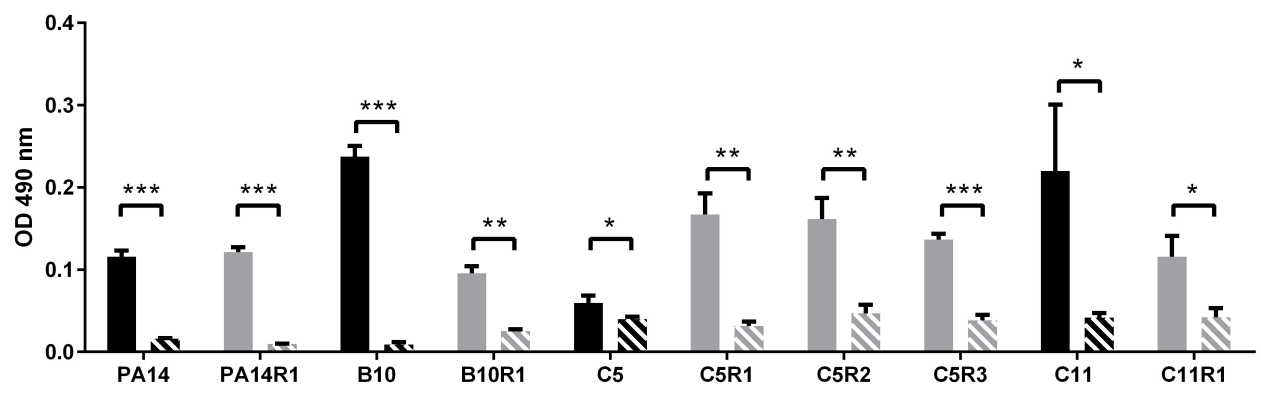

C

Pyocyanin production

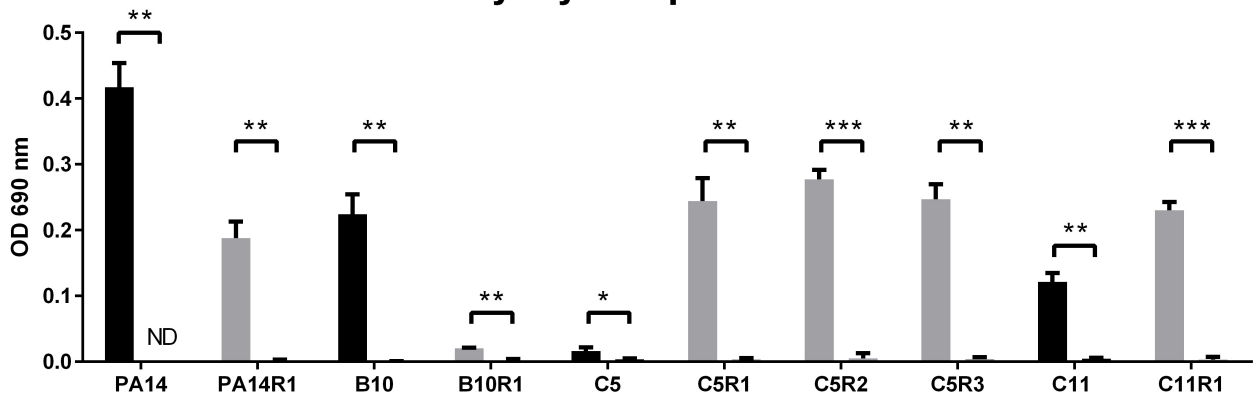

D

Biofilm formation

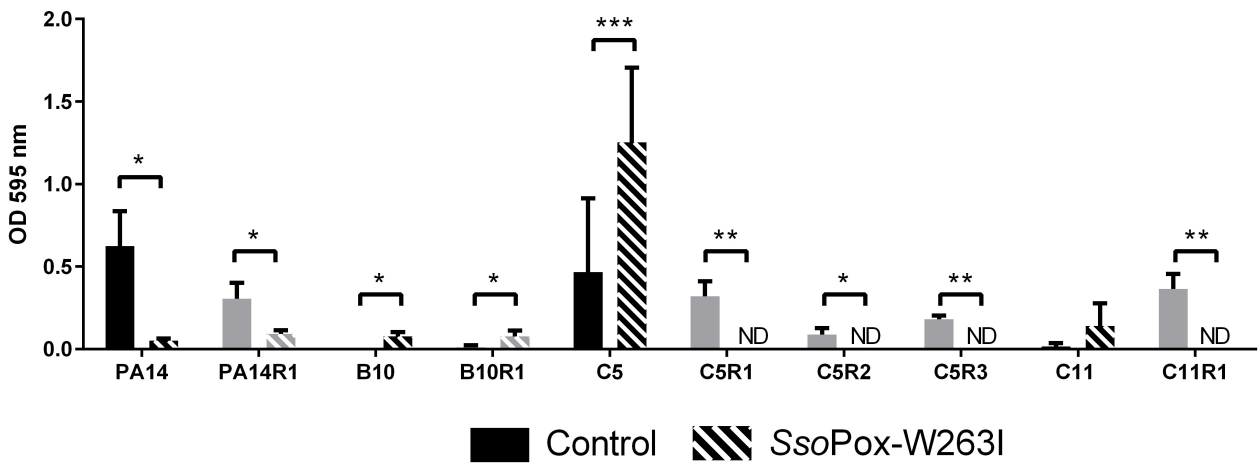

FIGURE 3 | Effect of SsoPox-W263l on virulence factors of phage resistant mutants (gray) and the associated parental strains (black) in vitro. For each strain, bars represent the mean of protease (A), elastase (B), pyocyanin (C) and biofilm (D) levels of three experiments for treated culture with $0.5 \mathrm{mg} \mathrm{ml}^{-1}$ SsoPox-W263l (striped bar) or inactive mutant 5A8 as negative control (empty bar). ND: not detected. Error bars represent the standard deviations of three experiments. ${ }^{*} p$-values $<0.05 ;{ }^{* *} p$-values $<0.01 ;{ }^{* * *} p$-values $<0.001$ according to Student's $t$-test. 
between the parental strains and their phage-resistant derivatives (Figure 3). It appears that the selection for phage-resistant clones did not result in the selection for mutants with only increased or only decreased biofilm formation. Similarly phageresistant mutants did not all increase nor decrease virulence factor secretion as compared to their parental strains. Thereby no common trade-off due to the selection of phage resistant bacteria can be drawn at the level of these four phenotypic traits. Altogether, these results show the efficiency of SsoPox-W263I in reducing the amount of three virulence factors characteristic of $P$. aeruginosa and modulating the production of biofilm in vitro in both antibiotic and phage-resistant isolates.

\section{Evaluation of Quorum Quenching in Amoeba, Virulence Model}

To further confirm the potential of SsoPox-W263I to decrease the virulence of bacterial isolates, the in vivo protecting effect of the enzyme was assayed using the amoeba A. polyphaga. The virulence of treated and control bacteria toward amoeba was assayed by measuring the propagation of $A$. polyphaga on a plate flooded by a pretreated bacterial lawn (Fenner et al., 2006).

Overall, SsoPox-W263I treatment decreased the virulence toward A. polyphaga of 9 out of 10 strains. No effect of QQ was observed for C11, for which virulence toward A. polyphaga remained unchanged with or without SsoPoxW263I treatment, but its phage resisting mutant C11R1 recovered a high sensitivity to the amoeba upon treatment (Figure 4). C5, C5R1, and PA14R1 were initially not virulent enough to prevent the propagation of amoeba in the control condition; however, treatment by SsoPox-W263I significantly enhanced its expansion (Figure 4). Consistently with in vitro observations on virulence factor production, the results obtained in vivo confirmed that parental and resistant strains behave differently, especially for PA14 and C5 (Figure 4). SsoPox-W263I treatment showed a benefic effect in all but one case and no negative effects were observed, highlighting the efficiency of the QQ treatment in reducing the virulence of antibiotic and phage resistant isolates in an in vivo model.

\section{Combined Effect of Enzymatic and Antimicrobial Treatment}

Considering that PA14 displayed antibiogram comparable to most clinical isolates tested in this study, PA14 and its phage resistant mutant PA14R1 were further used as representative candidates to evaluate the combined effect of SsoPox-W263I and antibiotic treatment using the amoeba infection model (Figure 5), then the combined effect of SsoPox-W263I and phage treatment was also assayed (Figure 6). As observed in our first experiment, with an enzyme concentration of $500 \mu \mathrm{g} \mathrm{ml}^{-1}$, QQ increases the sensitivity toward amoeba. Hence, to better assay the combined effect of enzymatic and antimicrobial treatments, lower doses of SsoPox-W263I were considered. Thus, following a dose response experiment on PA14, $10 \mu \mathrm{g} \mathrm{ml}^{-1}$ of SsoPoxW263I was used with antimicrobial as it had a lower impact on the virulence (Supplementary Figure 2).
Synergistic effect was observed with a treatment of SsoPoxW263I and $25 \mathrm{mg} \mathrm{ml}^{-1}$ ciprofloxacin on PA14, PA14R1 (Figure 5). At these concentrations, each treatment alone had no effect on the virulence of PA14 against the amoeba and the amoeba could not grow. However, with the combined treatment (ciprofloxacin + SsoPox-W263I), the amoeba was able to completely colonize the Petri dish in 6 and 7 days, respectively, for PA14. PA14R1 virulence was slightly impacted by the antibiotic treatment alone or the QQE treatment alone, but the highest effect was observed with the combined treatment: the Petri dish limit $(8.5 \mathrm{~cm})$ was reached 1 day earlier than with the QQE treatment alone, and the growth of amoeba started after 1 day with the combined treatment against 4 days with ciprofloxacin alone.

As the composition of the commercial cocktail was toxic for amoeba, PA14 lytic phages were purified from the cocktail and concentrated to $10^{8} \mathrm{PFU} \mathrm{ml} \mathrm{ml}^{-1}$. To test the combined effect of SsoPox-W263I and phages, ФIntesti-PA14 was used alone or in combination with the enzyme against PA14 and PA14R1. The isolated phages did not impact the growth of the amoeba. For both PA14 and PA14R1, amoeba growth was faster with SsoPoxW263I alone, yet the combined treatment led to a further increase of amoeba growth (Figure 6).

\section{DISCUSSION}

In this study, four strains of $P$. aeruginosa were used, including three clinical isolates from diabetic foot infections. The antibiotic resistance profiles of the strains revealed that all the clinical isolates presented a significant tolerance to rifampicin, trimethoprim/sulfamethoxazole and nitrofurantoin, confirming previous observations regarding the increasing rate of multi-resistance in diabetic foot infections in the recent years (Lipsky, 2016).

To address antibioresistance issues, bacteriophages and QQ have emerged as promising therapeutic approaches. As with antibiotics, the use of bacteriophages suffers from rapid resistance phenomena such as the formation of a biofilm, the modification of phage receptor expression that can reduce phage entry (Chapman-McQuiston and $\mathrm{Wu}, 2008$ ), or the cell adaptive inducible CRISPR-Cas (clustered regularly interspaced short palindromic repeat and CRISPR associated proteins) system that recognizes and degrades phage DNA (Barrangou et al., 2007). Interestingly, it has been extensively demonstrated that QQ reduces biofilm formation in $P$. aeruginosa, thereby increasing antimicrobial treatment efficacy, and a recent study has underlined that QS disruption can also decrease phage resistance by inhibiting QS stimulation of the CRISPR-Cas system (Høyland-Kroghsbo et al., 2017). The efficacy of SsoPox-W263I to modulate CRISPR-Cas system regulation of proteobacteria, including $P$. aeruginosa, was recently demonstrated (Mion et al., 2019). In addition, recent studies have shown that even without involvement of CRISPR-Cas defense, the phage infection outcome could be different in QS-deficient mutant of $P$. aeruginosa than in wild type strains (Qin et al., 2017; SaucedoMora et al., 2017). As antibiotic or phage resistance can induce 


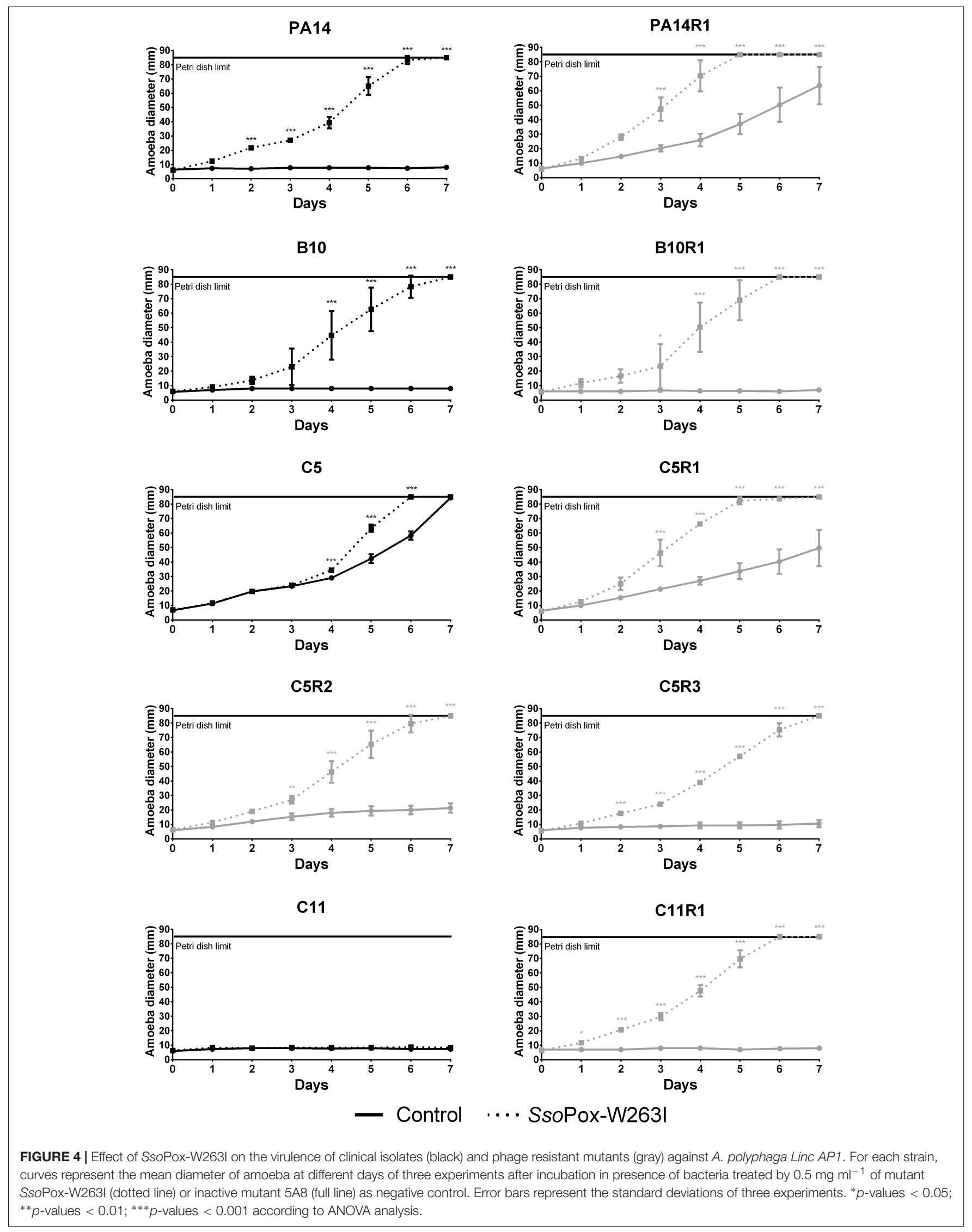




\section{PA14}

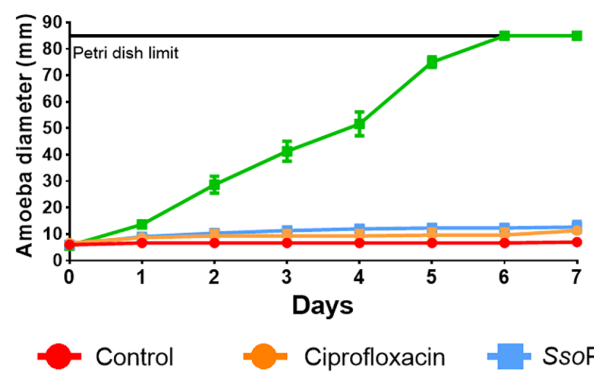

PA14R1

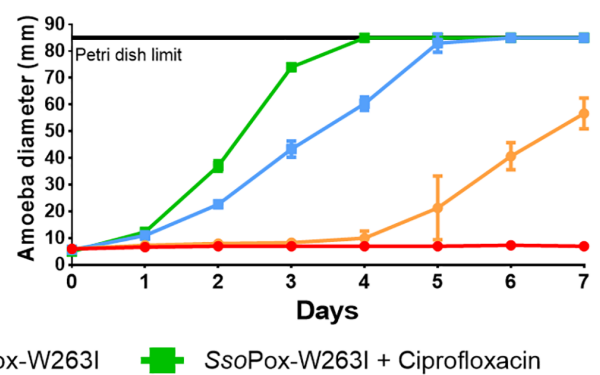

FIGURE 5 | Combinatory effect of SsoPox-W263I and ciprofloxacin on the virulence of clinical isolates and phage resistant mutants against $A$. polyphaga Linc AP1. For each strain, curves represent the mean diameter of amoeba at different days after incubation in presence of bacteria without treatment (red) or treated with

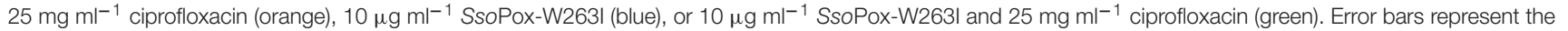
standard deviations of three experiments.

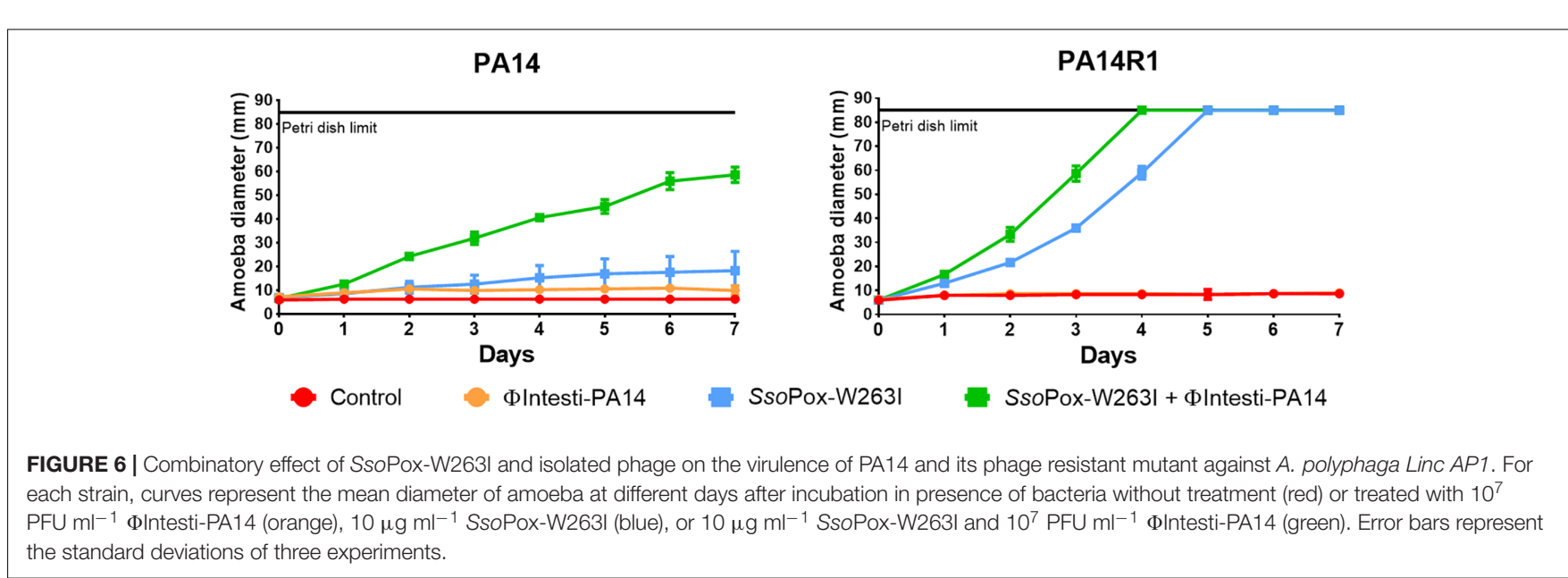

life-threatening complications, we evaluated the potential of QQ to act as an alternative therapeutic approach.

First, we selected six mutants derived from the four initial strains as phage resistant toward a commercial phage cocktail. The virulence profiles of each strain were then evaluated and the efficacy of three therapeutic approaches (antibiotics with ciprofloxacin, bacteriophages and QQ with SsoPoxW263I) on the different strains was further assessed alone or in combinations.

In several cases bacteriophage resistance did not affect antibiotic resistance, although the selection pressure induced by the phage cocktail resulted in a loss of resistance to ciprofloxacin and doxycycline in the mutant strains isolated from C5. This evolutionary trade-off is coherent with a previous study, where the phage OMKO1 which targets OprM, the porin of a multi-drug efflux system of $P$. aeruginosa as receptor-binding site, selected resistant bacteria harboring a change in the efflux mechanism with increased sensitivity to different antibiotic classes, such as tetracycline and fluoroquinolone (Chan et al., 2016).

To determine the potential of QQ treatment on the different strains, four QS-regulated traits, biofilm formation and the production of three virulence factors (pyocyanin, protease and elastase) were measured in vitro. As reported in other studies, some phage resistant bacteria such as C5R1, C5R2, and C5R3 exhibited higher level of virulence factor production than the initial strain (Hosseinidoust et al., 2013). The ability of SsoPox-W263I as QQ agent to decrease virulence in the different strains was also assayed. The QQ treatment significantly reduced the production of the virulence factors for the initial strains as well as their phage resistant mutants, showing that bacteria resistant to phage treatment conserved a functional QS system which can be inhibited by enzyme-mediated QQ. Strains harboring a higher virulence profile were also efficiently quenched using SsoPox-W263I. In order to correlate in vitro production of virulence factors, biofilm formation and in vivo virulence, an amoeba-based assay was developed. Amoeba are eukaryotic organisms able to feed on bacteria. Their phagocytosis and digestion mechanisms are similar to those of macrophage bacterial elimination (Greub and Raoult, 2004), thus amoeba are frequently used to test in vivo virulence of bacteria (Rémy et al., 2018). The model of A. polyphaga feeding on $P$. aeruginosa, where the growth of the amoeba directly indicates the pathogenicity level of the bacteria, was chosen to assess the efficiency of QQ treatment with SsoPox-W263I. The results showed that the inhibition of virulence by the QQ treatment was efficient for 9 out of 10 strains, allowing the 
growth of the amoeba, independently from the resistance profiles to antibiotics or bacteriophages. Interestingly, $P$. aeruginosa was previously proved to use type III secretion system (T3SS) to kill biofilm-associated amoebae potentially suggesting that differences observed upon enzymatic treatment could be related in the modification of T3SS regulation (Matz et al., 2008). Although variable enzyme effects on virulence factor production or biofilm formation were observed in vitro depending on the strains, our results indicate that QQ is efficient in vivo on most of the antibiotic or phage resistant clinical isolates. This highlights the potential of enzymatic QQ treatment as an interesting alternative in case of therapeutic dead ends.

Furthermore, we evaluated the potential of SsoPox-W263I to act as a complement of antibiotics or bacteriophages to counteract $P$. aeruginosa virulence toward A. polyphaga. Using lower concentrations of SsoPox-W263I, the combination of QQ and ciprofloxacin enhanced the growth of the amoeba for ciprofloxacin-sensitive strains PA14 and PA14R1 as compared to the antibiotic or the enzymatic treatment alone. Consistently with previous reports using the lactonase from Bacillus sp. ZA12 with ciprofloxacin in a murine burn infection model (Gupta et al., 2015), our results show that enzymatic QQ works in synergy with fluoroquinolones in sensitive strains decreasing efficiently the amount of antibiotics required to fight bacterial infections.

In addition to antibiotics, the synergy of SsoPox-W263I with bacteriophages was underlined. Synergistic effects were observed when $P$. aeruginosa PA14 was treated by the combined actions of isolated phage Intesti-PA14 and SsoPox-W263I. Interestingly, the synergy was also observed on PA14R1 strain, which was resistant to the phage cocktail. In concordance with these observations, recent reports showed close relationships between QS and phage tolerance mechanisms in P. aeruginosa (Mion et al., 2018).

Altogether, the results obtained in vitro and in vivo show that SsoPox-W263I is efficient to decrease bacterial virulence in model and clinical isolates of $P$. aeruginosa and constitute a proof of concept suggesting that enzymatic QQ can strengthen the therapeutic arsenal available against $P$. aeruginosa infections by enhancing the efficiency of available treatments including bacteriophages or antibiotics. In addition, it has been shown that this enzyme, issued from an extremophilic organism, resists harsh industrial conditions (Rémy et al., 2016) confirming its tremendous potential for biopharmaceutical applications and

\section{REFERENCES}

Barrangou, R., Fremaux, C., Deveau, H., Richards, M., Boyaval, P., Moineau, S., et al. (2007). CRISPR provides acquired resistance against viruses in prokaryotes. Science 315, 1709-1712. doi: 10.1126/science.11 38140

Bassler, B. L., and Losick, R. (2006). Bacterially speaking. Cell 125, 237-246. doi: 10.1016/j.cell.2006.04.001

Breidenstein, E. B. M., de la Fuente-Núñez, C., and Hancock, R. E. W. (2011). Pseudomonas aeruginosa: all roads lead to resistance. Trends Microbiol. 19, 419-426. doi: 10.1016/j.tim.2011.04.005

Chan, B. K., Sistrom, M., Wertz, J. E., Kortright, K. E., Narayan, D., and Turner, P. E. (2016). Phage selection restores antibiotic sensitivity in MDR Pseudomonas aeruginosa. Sci. Rep. 6:26717. doi: 10.1038/srep26717 should now be evaluated on mammalian models in order to reach clinical trials.

\section{DATA AVAILABILITY}

The datasets generated for this study are available on request to the corresponding author.

\section{AUTHOR CONTRIBUTIONS}

$\mathrm{SM}, \mathrm{BR}, \mathrm{LP}, \mathrm{FB}, \mathrm{DD}$, and EC designed the study and wrote the manuscript. SM, BR, and LP performed the experiments. SM, BR, LP, and DD analyzed the data.

\section{FUNDING}

$\mathrm{SM}$ is a Ph.D. student granted by the Direction Générale de l'Armement (DGA). BR received a $\mathrm{Ph} . \mathrm{D}$ grant from the "Emplois Jeunes Doctorants" program of Région ProvenceAlpes-Côte d'Azur (PACA, France). This work was supported by Investissements d'avenir program (Méditerranée Infection 10-IAHU-03) of the French Agence Nationale de la Recherche (ANR). This work also received support from RAPID (LACTO-TEX) program from the Direction Générale de l'Armement (DGA).

\section{ACKNOWLEDGMENTS}

We thank Dr. M. Ansaldi and Dr. P. Masson for providing phages; Dr. G. Dubourg for the antibiogram experiment; Prof. B. La Scola for providing amoeba and Prof. J-P Lavigne for providing strains. We also thank Dr. Mikael Elias for fruitful discussions.

\section{SUPPLEMENTARY MATERIAL}

The Supplementary Material for this article can be found online at: https://www.frontiersin.org/articles/10.3389/fmicb. 2019.02049/full\#supplementary-material

Chapman-McQuiston, E., and Wu, X. L. (2008). stochastic receptor expression allows sensitive bacteria to evade phage attack. part I: experiments. Biophys. J. 94, 4525-4536. doi: 10.1529/biophysj.107.120212

Chessa, J.-P., Petrescu, I., Bentahir, M., Van Beeumen, J., and Gerday, C. (2000). Purification, physico-chemical characterization and sequence of a heat labile alkaline metalloprotease isolated from a psychrophilic Pseudomonas species. Biochim. Biophys. 1479, 265-274. doi: 10.1016/S0167-4838(00)00018-2

Diop, A., Khelaifia, S., Armstrong, N., Labas, N., Fournier, P.-E., Raoult, D., et al. (2016). Microbial culturomics unravels the halophilic microbiota repertoire of table salt: description of Gracilibacillus massiliensis sp. nov. Microb. Ecol. Health Dis. 27:32049. doi: 10.3402/mehd.v27.32049

Domingo-Calap, P., Georgel, P., and Bahram, S. (2016). Back to the future: bacteriophages as promising therapeutic tools. HLA 87, 133-140. doi: 10.1111/ $\tan .12742$ 
Driscoll, J. A., Brody, S. L., and Kollef, M. H. (2007). The epidemiology, pathogenesis and treatment of Pseudomonas aeruginosa infections. Drugs 67, 351-368. doi: 10.2165/00003495-200767030-3

Ertugrul, B. M., Oncul, O., Tulek, N., Willke, A., Sacar, S., Tunccan, O. G., et al. (2012). A prospective, multi-center study: factors related to the management of diabetic foot infections. Eur. J. Clin. Microbiol. Infect. Dis. 31, 2345-2352. doi: 10.1007/s10096-012-1574-1

European Society of Clinical Microbiology and Infectious Diseases, (2018). Available at: http://www.eucast.org/clinical_breakpoints/ (accessed September 26, 2018).

Fenner, L., Richet, H., Raoult, D., Papazian, L., Martin, C., and La Scola, B. (2006). Are clinical isolates of Pseudomonas aeruginosa more virulent than hospital environmental isolates in amebal co-culture test? Crit. Care Med. 34, 823-828. doi: 10.1097/01.CCM.0000201878.51343.F1

Greub, G., and Raoult, D. (2004). Microorganisms resistant to free-living amoebae. Clin. Microbiol. Rev. 17, 413-433. doi: 10.1128/CMR.17.2.413-433.2004

Guendouze, A., Plener, L., Bzdrenga, J., Jacquet, P., Rémy, B., Elias, M., et al. (2017). Effect of quorum quenching lactonase in clinical isolates of Pseudomonas aeruginosa and comparison with quorum sensing inhibitors. Front. Microbiol. 8:277. doi: 10.3389/fmicb.2017.00227

Gupta, P., Chhibber, S., and Harjai, K. (2015). Efficacy of purified lactonase and ciprofloxacin in preventing systemic spread of Pseudomonas aeruginosa in murine burn wound model. Burns 41, 153-162. doi: 10.1016/j.burns.2014. 06.009

Hiblot, J., Gotthard, G., Chabriere, E., and Elias, M. (2012). Characterisation of the organophosphate hydrolase catalytic activity of SsoPox. Sci. Rep. 2:779. doi: 10.1038/srep00779

Hiblot, J., Gotthard, G., Elias, M., and Chabriere, E. (2013). Differential active site loop conformations mediate promiscuous activities in the lactonase SsoPox. PLoS One 8:e75272. doi: 10.1371/journal.pone.0075272

Hosseinidoust, Z., van de Ven, T. G. M., and Tufenkji, N. (2013). Evolution of Pseudomonas aeruginosa virulence as a result of phage predation. Appl. Environ. Microbiol. 79, 6110-6116. doi: 10.1128/AEM.01421-3

Høyland-Kroghsbo, N. M., Paczkowski, J., Mukherjee, S., Broniewski, J., Westra, E., Bondy-Denomy, J., et al. (2017). Quorum sensing controls the Pseudomonas aeruginosa CRISPR-Cas adaptive immune system. Proc. Natl. Acad. Sci. U.S.A. 114, 131-135. doi: 10.1073/pnas.1617415113

Hraiech, S., Hiblot, J., Lafleur, J., Lepidi, H., Papazian, L., Rolain, J.-M., et al. (2014). Inhaled lactonase reduces Pseudomonas aeruginosa quorum sensing and mortality in rat pneumonia. PLoS One 9:e107125. doi: 10.1371/journal.pone. 0107125

Jault, P., Leclerc, T., Jennes, S., Pirnay, J. P., Que, Y.-A., Resch, G., et al. (2018). Efficacy and tolerability of a cocktail of bacteriophages to treat burn wounds infected by Pseudomonas aeruginosa (PhagoBurn): a randomised, controlled, double-blind phase 1/2 trial. Lancet Infect. Dis. 19, 35-45. doi: 10.1016/S14733099(18)30482-1

Kakasis, A., and Panitsa, G. (2018). Bacteriophage therapy as an alternative treatment for human infections. A comprehensive review. Int. J. Antimicrob. Agents 53, 16-21. doi: 10.1016/j.ijantimicag.2018.09.004

Kropinski, A. M., Mazzocco, A., Waddell, T. E., Lingohr, E., and Johnson, R. P. (2009). Enumeration of bacteriophages by double agar overlay plaque assay. Methods Mol. Biol. 501, 69-76. doi: 10.1007/978-1-60327-164-6_7

Kutter, E., De Vos, D., Gvasalia, G., Alavidze, Z., Gogokhia, L., Kuhl, S., et al. (2010). Phage therapy in clinical practice: treatment of human infections. Curr. Pharm. Biotechnol. 11, 69-86. doi: 10.2174/138920110790725401

Labrie, S. J., Samson, J. E., and Moineau, S. (2010). Bacteriophage resistance mechanisms. Nat. Rev. Microbiol. 8, 317-327. doi: 10.1038/nrmicro2315

Lipsky, B. A. (2016). Diabetic foot infections: current treatment and delaying the 'post-antibiotic era.'. Diabetes Metab. Res. Rev. 32, 246-253. doi: 10.1002/dmrr. 2739

Loc-Carrillo, C., and Abedon, S. T. (2011). Pros and cons of phage therapy. Bacteriophage 1, 111-114. doi: 10.4161/bact.1.2.14590

Matz, C., Moreno, A. M., Alhede, M., Manefield, M., Hauser, A. R., Givskov, M., et al. (2008). Pseudomonas aeruginosa uses type III secretion system to kill biofilm-associated amoebae. ISME J. 2, 843-852. doi: 10.1038/ismej.2008.47

Mion, S., Plener, L., Rémy, B., Daudé, D., and Chabrière, É. (2019). Lactonase SsoPox modulates CRISPR-Cas expression in gram-negative proteobacteria using AHL-based quorum sensing systems. Res. Microbiol. doi: 10.1016/j. resmic.2019.06.004 [Epub ahead of print].
Mion, S., Rémy, B., Plener, L., Chabrière, E., and Daudé, D. (2018). Empêcher les bactéries de communiquer: diviser pour mieux soigner. Ann. Pharm. Fr. 76, 249-264. doi: 10.1016/j.pharma.2018.02.004

Price-Whelan, A., Dietrich, L. E. P., and Newman, D. K. (2007). Pyocyanin alters redox homeostasis and carbon flux through central metabolic pathways in Pseudomonas aeruginosa PA14. J. Bacteriol. 189, 6372-6381. doi: 10.1128/JB. 00505-7

Qin, X., Sun, Q., Yang, B., Pan, X., He, Y., and Yang, H. (2017). Quorum sensing influences phage infection efficiency via affecting cell population and physiological state. J. Basic Microbiol. 57, 162-170. doi: 10.1002/jobm. 201600510

Rémy, B., Mion, S., Plener, L., Elias, M., Chabrière, E., and Daudé, D. (2018). Interference in bacterial quorum sensing: a biopharmaceutical perspective. Front. Pharmacol. 9:203. doi: 10.3389/fphar.2018.00203

Rémy, B., Plener, L., Poirier, L., Elias, M., Daudé, D., and Chabrière, E. (2016). Harnessing hyperthermostable lactonase from Sulfolobus solfataricus for biotechnological applications. Sci. Rep. 6:37780. doi: 10.1038/srep37780

Rhoads, D. D., Wolcott, R. D., Kuskowski, M. A., Wolcott, B. M., Ward, L. S., and Sulakvelidze, A. (2009). Bacteriophage therapy of venous leg ulcers in humans: results of a phase I safety trial. J. Wound Care 18, 237-243. doi: 10.12968/jowc. 2009.18.6.42801

Rolain, J.-M., Hraiech, S., and Bregeon, F. (2015). Bacteriophage-based therapy in cystic fibrosis-associated Pseudomonas aeruginosa infections: rationale and current status. Drug Des. Devel. Ther. 9, 3653-3663. doi: 10.2147/DDDT. S53123

Salmond, G. P. C., and Fineran, P. C. (2015). A century of the phage: past, present and future. Nat. Rev. Microbiol. 13, 777-786. doi: 10.1038/nrmicro3564

Saucedo-Mora, M. A., Castañeda-Tamez, P., Cazares, A., Pérez-Velázquez, J., Hense, B. A., Cazares, D., et al. (2017). Selection of functional quorum sensing systems by lysogenic bacteriophages in Pseudomonas aeruginosa. Front. Microbiol. 8:1669. doi: 10.3389/fmicb.2017.01669

Smith, K. M., Bu, Y., and Suga, H. (2003). Induction and inhibition of Pseudomonas aeruginosa quorum sensing by synthetic autoinducer analogs. Chem. Biol. 10, 81-89. doi: 10.1016/S1074-5521(03)00002-4

Soyza, A. D., Hall, A. J., Mahenthiralingam, E., Drevinek, P., Kaca, W., DrulisKawa, Z., et al. (2013). Developing an international Pseudomonas aeruginosa reference panel. MicrobiologyOpen 2, 1010-1023. doi: 10.1002/mbo3.141

Stepanović, S., Vuković, D., Dakić, I., Savić, B., and Švabić-Vlahović, M. (2000). A modified microtiter-plate test for quantification of staphylococcal biofilm formation. J. Microbiol. Methods 40, 175-179. doi: 10.1016/S0167-7012(00) 00122-6

Suttle, C. A. (2005). Viruses in the sea. Nature 437:356. doi: 10.1038/nature04160 Tacconelli, E., Carrara, E., Savoldi, A., Harbarth, S., Mendelson, M., Monnet, D. L., et al. (2018). Discovery, research, and development of new antibiotics: the WHO priority list of antibiotic-resistant bacteria and tuberculosis. Lancet Infect. Dis. 18, 318-327. doi: 10.1016/S1473-3099(17)30753-3

Welsh, M. A., and Blackwell, H. E. (2016). Chemical genetics reveals environmentspecific roles for quorum sensing circuits in Pseudomonas aeruginosa. Cell Chem. Biol. 23, 361-369. doi: 10.1016/j.chembiol.2016.01.006

Wright, A., Hawkins, C. H., Änggård, E. E., and Harper, D. R. (2009). A controlled clinical trial of a therapeutic bacteriophage preparation in chronic otitis due to antibiotic-resistant Pseudomonas aeruginosa; a preliminary report of efficacy. Clin. Otolaryngol. 34, 349-357. doi: 10.1111/j.1749-4486.2009.01973.x

Conflict of Interest Statement: EC has a patent WO2014167140 A1 licensed to Gene\&GreenTK. BR, LP, DD, and EC report personal fees from Gene\&GreenTK during the conduct of the study.

The remaining authors declare that the research was conducted in the absence of any commercial or financial relationships that could be construed as a potential conflict of interest.

Copyright (C) 2019 Mion, Rémy, Plener, Brégeon, Chabrière and Daudé. This is an open-access article distributed under the terms of the Creative Commons Attribution License (CC BY). The use, distribution or reproduction in other forums is permitted, provided the original author(s) and the copyright owner(s) are credited and that the original publication in this journal is cited, in accordance with accepted academic practice. No use, distribution or reproduction is permitted which does not comply with these terms. 